\title{
Hole transport in porphyrin thin films
}

\author{
Tom J. Savenije ${ }^{1}$ and Albert Goossens ${ }^{2}$ \\ ${ }^{1}$ Laboratory for Inorganic Chemistry, Delft University of Technology, Julianalaan 136, 2628 BL Delft, The Netherlands \\ ${ }^{2}$ Radiation Chemistry Department, IRI, Delft University of Technology, Mekelweg 15, 2629 JB, Delft, The Netherlands
}

(Received 8 January 2001; published 31 August 2001)

\begin{abstract}
Hole transport in p-type organic semiconductors is a key issue in the development of organic electronic devices. Here the diffusion of holes in porphyrin thin films is investigated. Smooth anatase $\mathrm{TiO}_{2}$ films are coated with an amorphous thin film of zinc-tetra(4-carboxyphenyl) porphyrin (ZnTCPP) molecules acting as sensitizer. Optical excitation of the porphyrin stimulates the injection of electrons into the conduction band of $\mathrm{TiO}_{2}$. The remaining holes migrate towards the back electrode where they are collected. Current-voltage and capacitance-voltage analysis reveal that the $\mathrm{TiO}_{2} / \mathrm{ZnTCPP}$ system can be regarded as an $n$ - $p$ heterojunction, with a donor density of $N_{D}=2.0 \times 10^{16} \mathrm{~cm}^{-3}$ for $\mathrm{TiO}_{2}$ and an acceptor density $N_{A}=4.0 \times 10^{17} \mathrm{~cm}^{-3}$ for ZnTCPP films. The acceptor density in porphyrin films increases to $1.3 \times 10^{18} \mathrm{~cm}^{-3}$ upon irradiation with $100-\mathrm{mW} \mathrm{cm}^{-2}$ white light. Intensity-modulated photocurrent spectroscopy, in which ac-modulated irradiation is applied, is used to measure the transit times of the photogenerated holes through the films. A reverse voltage bias hardly affects the transit time, whereas a small forward bias yields a decrease of the transit time by two orders of magnitude. Application of background irradiation also reduces the transit time considerably. These observations are explained by the presence of energy fluctuation of the highest-occupied molecular orbital level in the porphyrin films due to a dispersed conformational state of the molecules in the amorphous films. This leads to energetically distributed hole traps. Under short circuit and reverse bias, photogenerated holes reside most of the time in deep traps and their diffusivity is only $7 \times 10^{-11} \mathrm{~cm}^{2} \mathrm{~s}^{-1}$. Deep traps are filled by application of a forward bias and by optical irradiation leading to reduction of the transit time and a concomitant increase of the diffusivity up to $2 \times 10^{-7} \mathrm{~cm}^{2} \mathrm{~s}^{-1}$.
\end{abstract}

DOI: 10.1103/PhysRevB.64.115323

PACS number(s): 73.61.-r, 72.40.+w, 71.35.-y

\section{INTRODUCTION}

Although sensitization of wide band-gap semiconductors with dye molecules, like porphyrines and phthalocyanines, has been studied extensively in the past, ${ }^{1,2}$ fundamental aspects of sensitization are still an important research topic. An efficient $(\sim 10 \%)$ solar cell based on sensitization of $\mathrm{TiO}_{2}$ has been realized by O'Regan and Grätzel in $1991 .^{3}$ They used nanoporous $\mathrm{TiO}_{2}$, grafted it with ruthenium (bipyridyl) complexes, and soaked it in an iodine/iodide redox electrolyte. To enhance the operating stability and ease of production, replacement of the liquid electrolyte in these cells is an important issue..$^{4-7}$ Therefore, one may consider a combined function of the dye molecules: as sensitizer and as holetransport medium. This requires a sensitizer in which strong optical absorption and a high hole mobility are combined. Here porphyrin thin films are investigated to explore whether this requirement can be fulfilled.

Not only ruthenium complexes are efficient sensitizers for $\mathrm{TiO}_{2}$ with quantum efficiencies reaching unity, ${ }^{3}$ put also porphyrins with specific side groups can be used. ${ }^{8-11}$ Ultraviolet photoelectron spectroscopy $^{12}$ and electrochemical ${ }^{11,13-15}$ studies show that porphyrin films can often be regarded as $p$-type semiconductors. Despite numerous studies focusing on this issue, ${ }^{13,16-27}$ hole transport in molecular thin films is not well understood. For porphyrins and related molecules, a large discrepancy exists between the reported mobilities derived from current-voltage, ${ }^{21,25,26}$ time-of-flight, ${ }^{16-18,22}$ and microwave-conductivity measurements. ${ }^{20}$ Some of the differences can be related to the different side groups of the porphyrins used in the different studies, by which the molecules are forced to adapt a specific orientation. Other factors are the presence of impurities or imperfections, like grain boundaries, which are introduced during deposition of the film. Electrodeless microwave techniques reveal high mobilities in well-ordered stacks of porphyrins. In current-voltage and time-of-flight techniques, hole migration over a macroscopic distance is involved leading to a lower hole mobility.

The presence of a strong internal electric field in the organic film may also affect the charge-transport properties. In a heterostructure of a $p$-type organic dye and an $n$-type material, a region depleted of mobile charge carriers is created resulting in a built-in electric field. In the presence of such a depletion layer, the doping concentrations and the built-in potential $V_{\mathrm{bi}}$ can be derived from: ${ }^{28}$

$$
\frac{1}{C^{2}}=\frac{2}{e \varepsilon_{a}}\left(\frac{1}{\varepsilon_{A} N_{A}}+\frac{1}{\varepsilon_{B} N_{B}}\right)\left(V+V_{\mathrm{bi}}\right),
$$

where $C$ is the capacitance per $\mathrm{m}^{2}, e$ the electronic charge, $\varepsilon_{O}$ the permitivity of free space, $\varepsilon_{A}, \varepsilon_{B}$ the dielectric constants of the $n$-type and $p$-type materials, $N_{A}, N_{B}$ the doping densities of $n$ - and $p$-type materials, and $V$ the applied voltage. For organic films the presence of a depletion region has been demonstrated by impedance spectroscopy. ${ }^{15,24,25,29}$ The internal electric field contributes to suppress recombination of injected electrons in $\mathrm{TiO}_{2}$ with holes in the highestoccupied molecular orbital (HOMO) of the dye molecule by accelerating their separation.

In the present investigations, smooth anatase $\mathrm{TiO}_{2}$ films deposited on tin-doped indium oxide (ITO) glass are covered with a thin film of dye molecules, i.e., zinc-tetra(4carboxyphenyl) porphyrin (ZnTCPP, Fig. 1), which is a stable and efficient sensitizer, ${ }^{8,10}$ and forms macroscopically 


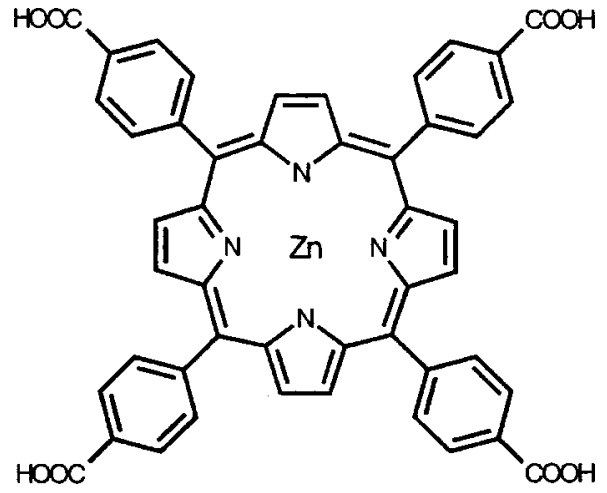

FIG. 1. Molecular structure of zinc-tetrakis(4-carboxyphenyl) porphyrin (ZnTCPP).

homogeneous amorphous films when using the spin-coating technique. From optical and electrochemical measurements ${ }^{8,11}$ the relevant energy levels of anatase $\mathrm{TiO}_{2}$ and porphyrin films have been derived and are shown in Fig. 2. Since the studied films are relatively thin, incident photons are absorbed throughout the film. However, previous studies of heterostructures with amorphous dye films have shown that only molecules in close proximity to the interface contribute to the photocurrent. ${ }^{15,30-33}$ This implies that excitons are not able to migrate over large distances ${ }^{14,33}$ and only those created near the interface between $\mathrm{TiO}_{2}$ and ZnTCPP dissociate into a conduction-band electron and a hole in the HOMO of ZnTCPP.

Eludication of hole transport in ZnTCPP thin films is the subject of the present paper and is a key issue in various types of organic solid-state devices. To examine whether a depletion layer is formed upon contacting $n$-type anatase $\mathrm{TiO}_{2}$ with a $p$-type $\mathrm{ZnTCPP}$, impedance spectroscopy is applied. To establish how the built-in electric field affects the transport of the holes in ZnTCPP films, the transit time is determined using intensity-modulated photocurrent spectroscopy. ${ }^{34,35}$

\section{EXPERIMENTAL ASPECTS}

Thin films $(\sim 200 \mathrm{~nm})$ of anatase $\mathrm{TiO}_{2}$ are deposited on ITO (30 $\Omega$ /square, Glastron) using metal-organic chemical-

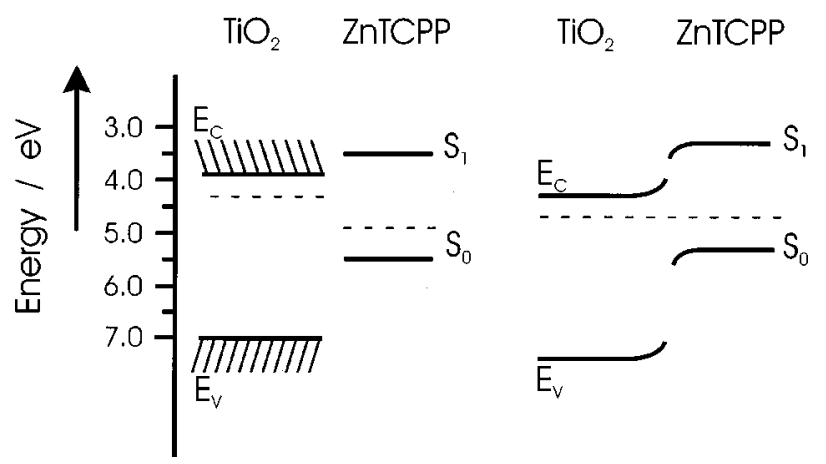

FIG. 2. Schematic one-electron energy diagrams of $n$-type anatase $\mathrm{TiO}_{2}$ and $p$-type $\mathrm{ZnTCPP}$ before contact (left) and after contact (right). vapor deposition to yield smooth and transparent layers as described previously. ${ }^{36}$ Onto these substrates, films of zinctetrakis(4, -carboxyphenyl) porphyrin (ZnTCPP, Fig. 1) with varying thicknesses are applied by spin coating from a 1-mM solution of ZnTCPP in methanol at spin velocities ranging from 1500 to $4000 \mathrm{rpm}$ under ambient conditions. This method yields homogeneous amorphous films with a reproducible thickness. The film thickness is derived from the absorbance, which is corrected for reflection using as extinction coefficient $\alpha=3.7 \times 10^{6} \mathrm{~m}^{-1}$ at $\lambda=563 \mathrm{~nm}$. ${ }^{37}$

A back contact is provided by a mercury droplet as described previously. ${ }^{32}$ Current vs voltage and capacitance vs voltage (Mott-Schottky) plots are recorded with an electrochemical interface (Solartron, Model 1286) in combination with a frequency-response analyzer (FRA) (Solartron, Model 1255). Photocurrent action spectra are recorded using a tungsten-halogen light source in combination with a monochromator. Intensity-modulated photocurrent spectroscopy (IMPS) spectra are recorded with the aid of a $2-\mathrm{mW}$ green $(\lambda=543.5 \mathrm{~nm})$ He:Ne laser modulated by a photoacoustic modulator (Isomet 1205C-2) driven by the generator output of the FRA. Additional bias light is provided by a $50-\mathrm{W}$ tungsten-halogen light source with a homogeneous intensity of $1000 \mathrm{~W} \mathrm{~m}^{-2}$. As reference a fast $\mathrm{Si}$ photodiode (Hammamatsu) is used, onto which a small fraction of the modulated light is directed. The photocurrent of the cell and the reference are measured across a $50 \Omega$ precision resistance and are identically amplified to eliminate phase shifts. From the ratio of these signals, the phase shift and the relative intensity of the ac photocurrent are derived, which yield admittance spectra for photocurrent generation. The admittance $Y(\omega)$ is defined as $i_{\mathrm{ph}}(\omega) / e \Phi(\omega)$ in which $\Phi(\omega)$ is the irradiation intensity in photons per second, which is a function of the modulation frequency and is composed of a dc and an ac part; $i_{\mathrm{ph}}(\omega)$ is the generated photocurrent, which also has dc and ac components. $Y(\omega)$ is conveniently treated as a complex function: $Y(\omega)=Y_{\text {Real }}(\omega)+i Y_{\text {Imag }}(\omega)$ in which $Y_{\text {Real }}(\omega)$ represents the in-phase signal and $Y_{\text {Imag }}(\omega)$, represents the out-of-phase signal. In the limit $\omega \rightarrow 0$ there is no phase shift; $Y_{\text {Imag }}(\omega=0)$ is zero and $Y_{\text {Real }}(\omega=0)$ is the external quantum efficiency. The photocurrent admittance $Y(\omega)$ can be modeled with an electrical equivalent circuit, which is a virtual electronic circuit with the same frequency response as the system under study. Nonlinear least-squares fitting is used to correlate the equivalent circuit to the IMPS spectra. Subsequently, time constants for the different processes in the cells are extracted. It should be noted that all the experiments are carried out under ambient conditions, which implies that an oxygen partial pressure of 0.2 bar is present.

\section{RESULTS AND DISCUSSION}

Current-voltage and capacitance-voltage plots

Current-voltage plots of an $\mathrm{ITO} / \mathrm{TiO}_{2} / \mathrm{ZnTCPP} / \mathrm{Hg}$ cell in the dark and under $100 \mathrm{~mW} \mathrm{~cm}^{-2}$ are shown in Fig. 3(a). For cells without a ZnTCPP film, the curves in the dark and under irradiation are identical; the open-circuit photovoltage 

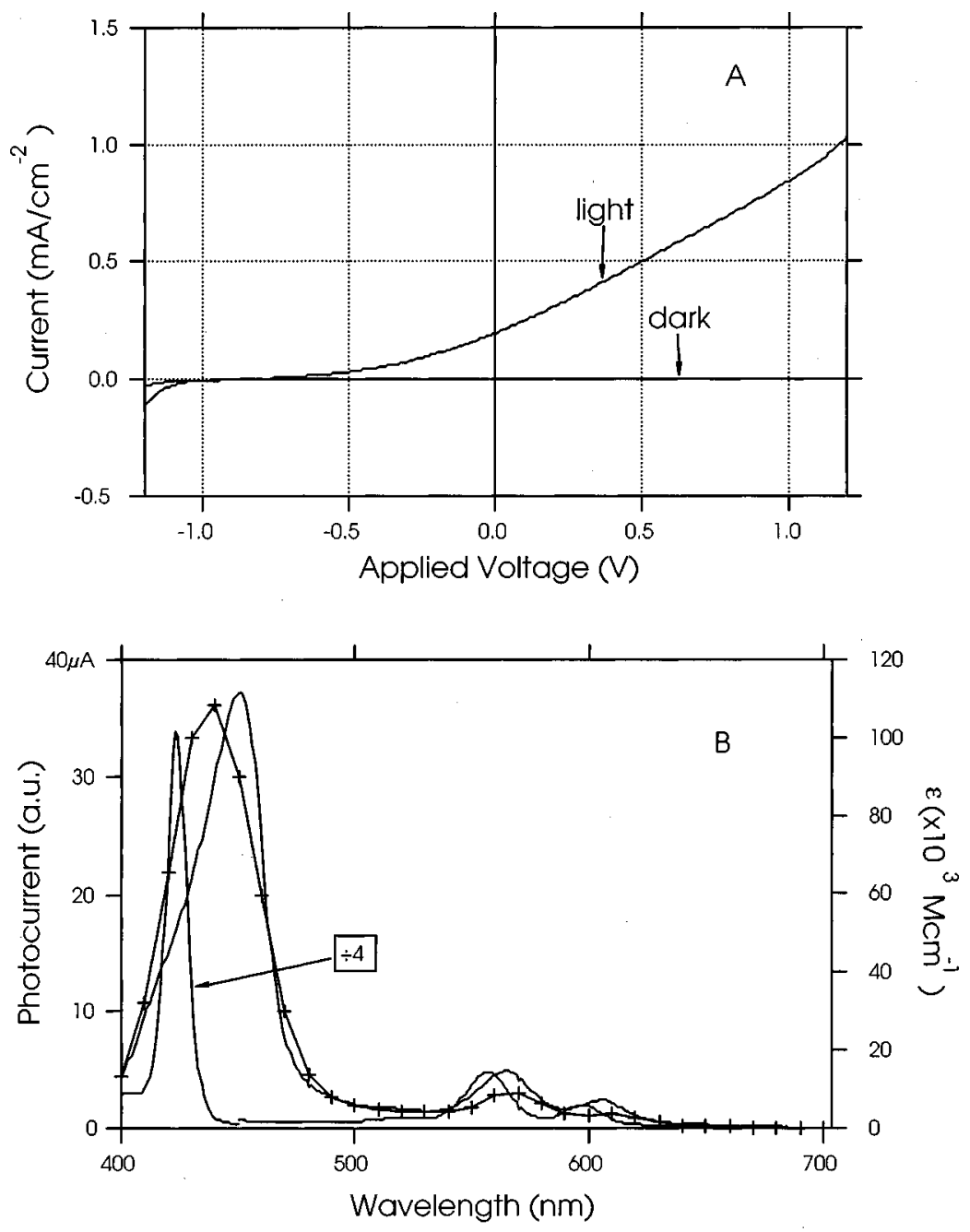

FIG. 3. (a) Current-voltage plots in the dark and under white-light irradiation (100 $\mathrm{mW} \mathrm{cm}{ }^{-2}$ ). (b) Photocurrent action spectra of an ITO/TiO $2(200 \mathrm{~nm}) / \mathrm{ZnTCPP}(\sim 32 \mathrm{~nm}) / \mathrm{Hg}$ device (dash). For comparison the absorption spectra of a spin-coated sample on quartz $(+++)$ and a $2-\mu \mathrm{M}$ ZnTCPP solution in methanol (-) are included. For clarity, the ZnTCPP methanol solution absorption spectrum below $450 \mathrm{~nm}$ is divided by 4 . The extinction coefficient for the film is normalized to the extinction coefficient of the solution at the maximum of the largest $Q$ band $(560 \mathrm{~nm})$.
$V_{\mathrm{OC}}$ and short circuit photocurrents $i_{\mathrm{SC}}$ are both zero. ITO/TiO $2 / \mathrm{ZnTCPP} / \mathrm{Hg}$ cells, however, show a $V_{\mathrm{OC}}$ of $0.7 \mathrm{~V}$ and an $i_{\mathrm{SC}}$ of $0.22 \mathrm{~mA} \mathrm{~cm}^{-2}$. The fill factor is 0.25 , which is typical for organic photovoltaic cells. ${ }^{14}$ Using thinner ZnTCPP films results in higher photocurrents, but simultaneously $V_{\mathrm{OC}}$ decreases probably due to electrical shorts as has been observed before. $^{38}$ Therefore, $\sim 30$-nm-thin ZnTCPP films have been used. The photocurrent action spectrum resembles the attenuation spectrum of the spin-coated film, which is defined as $1-I_{t} / I_{o}$ in which $I_{t}$ is the transmitted light intensity and $I_{o}$ is the incident light intensity. Since the optical density has a maximum of 0.3 the absorption and attenuation spectra are almost identical. In Fig. 3(b) the photocurent action spectrum and the absorption spectrum of the porphyrin films are compared. At wavelengths below $400 \mathrm{~nm}$, absorption by anatase $\mathrm{TiO}_{2}$ starts to contribute to the photocurrent.

Impedance spectra in the dark are presented in Fig. 4(a) and show distorted semicircles between $2 \mathrm{~Hz}$ and $200 \mathrm{kHz}$. They can be fitted to an equivalent circuit with a parallel resistor and a constant phase element (CPE) in series with a second resistor. The impedance of a CPE is $Q^{-1}(j \omega)^{-n}$; for $n=0$ it resembles a resistance and for $n=1$ it resembles a capacitance. Intermediate values of $n$ indicate dispersive charging processes. The frequency response at $10 \mathrm{kHz}$ and higher can be approximated by an equivalent circuit with a resistor and a capacitor connected in series. Figure 4(b) shows the reciprocal of the square of the capacitance vs voltage (Mott-Schottky plots) at $10 \mathrm{kHz}$ at different light intensities. Three distinct regimes are observed of which only one varies with the light intensity, as is further discussed below.

To interpret the impedance spectra and the Mott-Schottky plots, first a cell without the ZnTCPP layer is considered (data not shown). As has been previously reported, ${ }^{39}$ an ITO/TiO $2 / \mathrm{Hg}$ cell shows rectification due to the formation of a Schottky barrier upon contacting $n$-type $\mathrm{TiO}_{2}$ with mercury. The $C^{-2}$ vs $V$ plot of this cell shows two different slopes, indicating the presence of a depleted region spread across two different semiconductors. By applying Eq. (1) two values for the product of the relative dielectric constant and doping density $\left(\varepsilon N_{D}\right)$ are found, i.e., $2.4 \times 10^{18}$ and $3.1 \times 10^{20} \mathrm{~cm}^{-3}$, which are assigned to $\mathrm{TiO}_{2}$ and ITO, respectively.

For $\mathrm{ITO} / \mathrm{TiO}_{2} / \mathrm{ZnTCPP} / \mathrm{Hg}$ devices three different slopes can be distinguished in the Mott-Schottky plot [Fig. 4(b), curve + ], and with the aid of Eq. (1)

$$
\left\{\left(\varepsilon_{A} N_{A}\right)^{-1}+\left(\varepsilon_{B} N_{B}\right)^{-1}\right\}^{-1}
$$



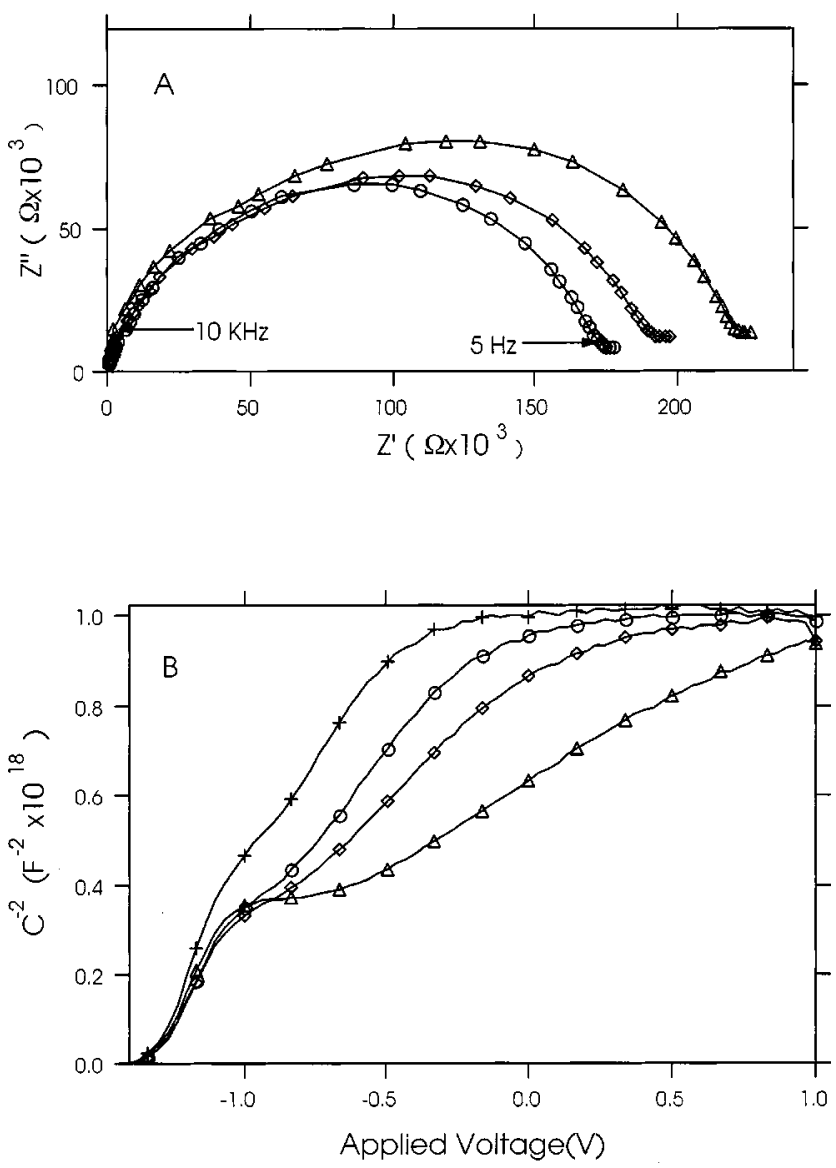

FIG. 4. (a) Electric impedance spectra of $\mathrm{ITO} / \mathrm{TiO}_{2} / \mathrm{ZnTCPP} / \mathrm{Hg}$ cells between $2 \mathrm{~Hz}$ and $200 \mathrm{kHz}$ with the real part of the impedance $Z^{\prime}$ on the horizontal axis and the imaginary part $Z^{\prime \prime}$ multiplied by -1 on the vertical axis. $V=0.5 \mathrm{~V}(\triangle), V=0 \mathrm{~V}(\diamond)$, and $V$ $=-0.3 \mathrm{~V}(\bigcirc)$ without light. (b) $C^{-2}$ vs $V$ (Mott-Schottky) plots recorded at $10 \mathrm{kHz}$ in the dark $(\triangle)$, under $1 \%(\diamond), 10 \%(\bigcirc)$, and $100 \%(+)$ of $100-\mathrm{mW} \mathrm{cm}^{-2}$ tungsten-halogen irradiation.

is found to be $1.0 \times 10^{18}, 1.8 \times 10^{18}$, and $1.2 \times 10^{20} \mathrm{~cm}^{-3}$ for the three regimes. Upon applying white light, only one of the three slopes, i.e., that between -0.8 and $-0.3 \mathrm{~V}$ is affected, indicating charging of the ZnTCPP film, since only this film absorbs visible light. Comparing the two other slopes with those of the $\mathrm{ITO} / \mathrm{TiO}_{2} / \mathrm{Hg}$ cells allows assignment of the $C^{-2}$ vs $V$ slope between -1.3 and $-1.0 \mathrm{~V}$ to the $\mathrm{TiO}_{2}$ film and the slope between -0.3 and $1.0 \mathrm{~V}$ to ITO. Substituting a relative dielectric constant of 55 for $\mathrm{TiO}_{2}$ and 4.5 for ZnTCPP (Ref. 40) yields $N_{D}=2.0 \times 10^{16} \mathrm{~cm}^{-3}$ for $\mathrm{TiO}_{2}$ and $N_{A}=4.0 \times 10^{17} \mathrm{~cm}^{-3}$ for ZnTCPP. Estimating a density of 1 $\mathrm{g} \mathrm{cm}^{-3}$ for porphyrin thin films shows that in the depletion region only 1 out of every 1000 molecules is oxidized. From the direction of the $i_{\mathrm{SC}}$ the sign of $V_{\mathrm{OC}}$ [Fig. 3(a)], and the Mott-Schottky plots [Fig. 4(b)], ITO/TiO $2 / \mathrm{ZnTCPP} / \mathrm{Hg}$ cells can be regarded as $n^{+}-n-p$ heterojunction devices with a highly doped $n^{+}$-type ITO film, an $n$-doped $\mathrm{TiO}_{2}$ film, and a $p$-doped ZnTCPP film.

The intercept of the Mott-Schottky plot with the potential axis equals $V_{\mathrm{bi}}$, which is $1.3 \mathrm{~V}$. This is close to the difference between the conduction band of the $\mathrm{TiO}_{2}(4.1 \mathrm{eV}$ vs vacuum) and the ionization potential of ZnTCPP (5.3 eV vs vacuum), ${ }^{8}$ which implies that the Fermi level of ZnTCPP films is located near the HOMO energy. The open-circuit voltage is 0.7 $\mathrm{V}$ [see Fig. 3(a)], which shows that $\mathrm{ITO} / \mathrm{TiO}_{2} / \mathrm{ZnTCPP}$ cells do not reach the flat -band situation when $100 \mathrm{~mW} \mathrm{~cm}^{-2}$ irradiation is applied. Since the porphyrin films are very thin, a small back current flows, which precludes saturation of $V_{\mathrm{OC}}$. Applying thicker films reduces the back flow and give higher photovoltages, but the short-circuit current diminishes due to an increased film resistance.

A reversible change of the slope of the Mott-Schottky plot between -0.8 and $-0.5 \mathrm{~V}$ upon illumination is observed. Substituting a dielectric constant of 4.5 in Eq. (1) the acceptor density is found to increase from $N_{A}=4.0 \times 10^{17} \mathrm{~cm}^{-3}$ in the dark to $N_{A}=6.1 \times 10^{17} \mathrm{~cm}^{-3}$ at $1 \%, 8.8 \times 10^{17} \mathrm{~cm}^{-3}$ at $10 \%$, and $1.3 \times 10^{18} \mathrm{~cm}^{-3}$ at $100 \%$ irradiation intensity. This increase is assigned to photodoping, as has been reported by Nevin et al. ${ }^{38}$ In their investigations porphyrin layers are treated with $I_{2}$ vapor. Excitation of porphyrin stimulates electron transfer from the LUMO to $I_{2}$, yielding oxidized porphyrin molecules, i.e., holes. Similar findings have been reported for phthalocyanine films. ${ }^{41}$ In our films, holes are probably formed due to electron transfer from photoexcited porphyrin to $\mathrm{O}_{2}$, which has redox properties comparable to $I_{2}$. In the ground state, $\mathrm{ZnTCPP}$ is coordinated with $\mathrm{O}_{2}$ to form a polarized complex that dissociates upon irradiation, i.e.,

$$
\begin{aligned}
\left(\mathrm{ZnTCPP}^{\delta+} \cdots \mathrm{O}_{2}^{\delta-}\right)+\text { energy } & \rightarrow\left(\mathrm{ZnTCPP}^{+}+\mathrm{O}_{2}^{-}\right)^{*} \\
& \rightarrow \mathrm{ZnTCPP}^{+}+\mathrm{O}_{2}^{-} .
\end{aligned}
$$

The required energy can be provided by direct excitation of the complex or by energy transfer from neighboring excited ZnTCPP molecules. Also electron transfer from ZnTCCP* to the complex is possible. The created metastable chargetransfer state involves the presence of fixed negative charges inside the film $\left(\mathrm{O}_{2}^{-}\right)$, i.e., acceptors and accompanying holes $\left(\mathrm{ZnTCPP}^{+}\right)$. This type of photodoping explains the observed changes in the Mott-Schottky plots due to irradiation.

The depletion width $W_{B}$ in the porphyrin film is given by

$$
w_{B}=\sqrt{2 \varepsilon_{0} \varepsilon_{B} \varepsilon_{A} N_{A}\left(V+V_{\mathrm{bi}}\right) / e N_{B}\left(\varepsilon_{A} N_{A}+\varepsilon_{B} N_{B}\right)}
$$

in which the subscripts $A$ are related to the $n$-type $\mathrm{TiO}_{2}$ and $B$ to the $p$-type ZnTCPP, and a similar equation holds for the depletion width in $\mathrm{TiO}_{2} \cdot{ }^{28}$ In the dark the depletion width in $\mathrm{TiO}_{2}$ amounts to $480 \mathrm{~nm}$; accordingly 200-nm-thin films are fully depleted at $0 \mathrm{~V}$ as is indeed observed [Fig. 4(b)]. For ZnTCPP the depletion width at $V=0 \mathrm{~V}$ is $24 \mathrm{~nm}$, which shows that at this potential $32-\mathrm{nm}$ films are almost completely depleted, in accordance with the observations. The depletion width in ZnTCPP diminishes on illumination since $N_{A}$ increases. At $100-\mathrm{mW} \mathrm{cm}^{-2}$ tungsten-halogen light, $N_{A}$ is increased to $1.3 \times 10^{18} \mathrm{~cm}^{-3}$, which corresponds to a depletion width at $V=0 \mathrm{~V}$ of $8.5 \mathrm{~nm}$. Full depletion is not reached at $V=0 \mathrm{~V}$, which is in line with the observations [see Fig. 4(b)]. 

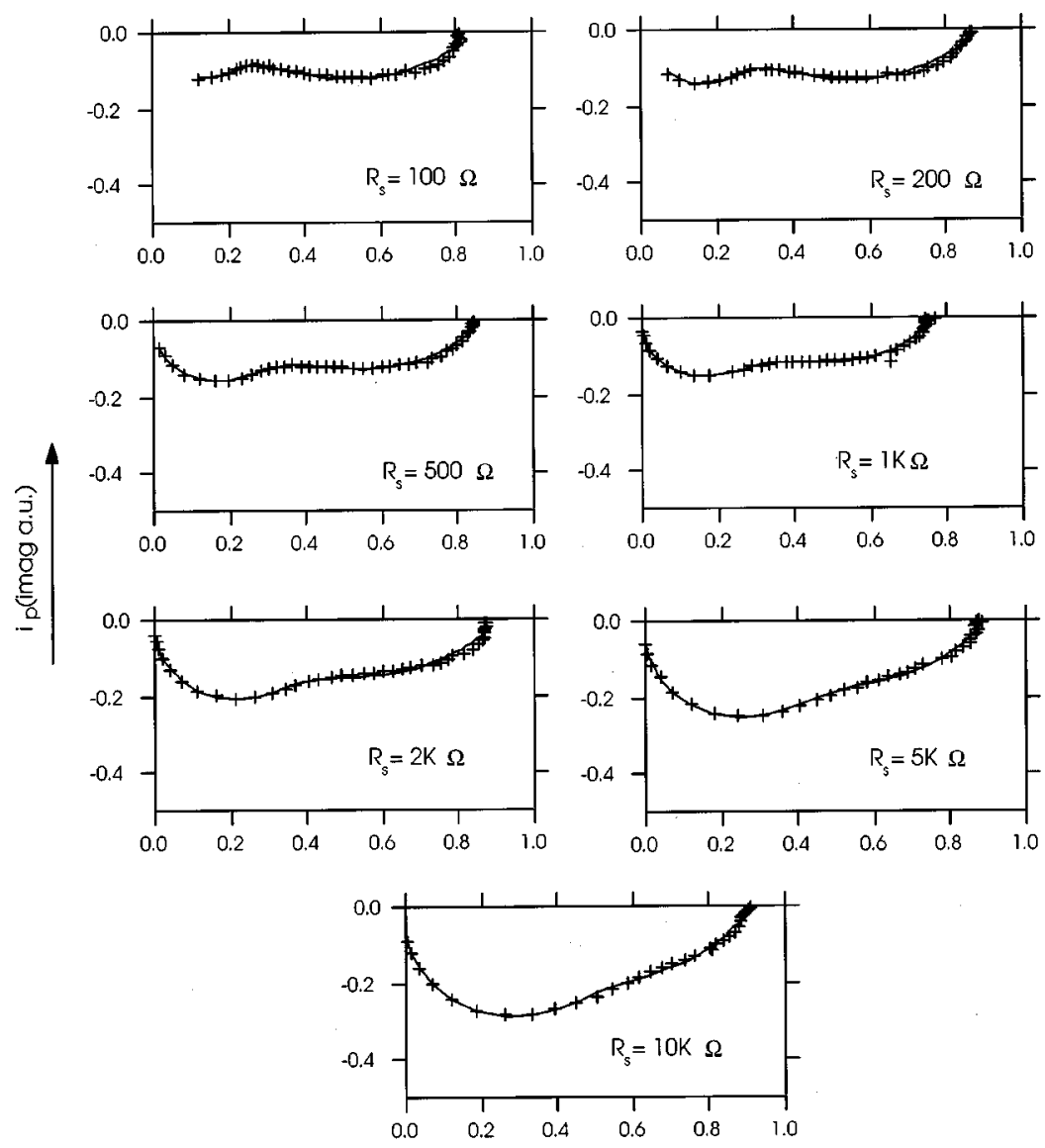

$I_{p}$ (real a.u.)

FIG. 5. Intensity-modulated photocurrent spectroscopy (IMPS) spectra (+) of $\mathrm{ITO} / \mathrm{TiO}_{2}$ $(200 \mathrm{~nm}) / \mathrm{ZnTCPP}(45 \mathrm{~nm}) / \mathrm{Hg}$ cells with an additional series resistor $R_{s}$ and without light at $V$ $=0 \mathrm{~V}$. The fits of the equivalent circuit to the IMPS spectra are excellent and are represented by the solid lines drawn through the data points. The fit parameters are collected in Table I. A 50 $\Omega$ resistor is used to measure the current.
Intensity-modulated photocurrent spectroscopy

Figure 5 shows a series of IMPS between $2 \mathrm{~Hz}$ and 200 $\mathrm{kHz}$. Two depressed semicircles are observed lying mainly in the fourth quadrant. When external resistances $R_{s}$ are connected in series with $\mathrm{ITO} / \mathrm{TiO}_{2} / \mathrm{ZnTCPP} / \mathrm{Hg}$ cells, the shape of the IMPS spectra changes. The intercept with the horizontal $Y_{\text {Real }}$ axis in Fig. 5 is related to the external quantum efficiency and is not affected by $R_{s}$. When the applied voltage is increased from -0.3 to $0.6 \mathrm{~V}, Y_{\text {Real }}(\omega=0)$ gradually increases either with or without background irradiation (Fig. 6). $Y_{\text {Real }}(\omega=0)$ reduces when background light is applied due to enhanced recombination of light-induced charge carriers at higher light intensities. Furthermore, at low potentials $\left(V_{a}<-0.2 \mathrm{~V}\right)$ and low frequencies, a response in the first quadrant is observed, which is also related to charge-carrier recombination. ${ }^{34}$

In order to unravel the different processes that are involved in ac photocurrent generation, IMPS spectra are modeled with an equivalent circuit. Combinations of circuit elements are related to different physical processes in the cells. The following processes must be considered: (i) charging of the capacitor formed by the geometry of the cell, (ii) transport of holes through the ZnTCPP, and (iii) recombination of optically generated charge carriers. Since recombination is dominant only at low voltages and at low frequencies the discussion is focused on processes (i) and (ii). Two characteristic time constants $\tau_{1}$ and $\tau_{2}$ are involved, one for the geometrical capacitance and one for hole transport. Accordingly, the IMPS spectra are fitted to an equivalent circuit consisting of two subcircuits in series. Each subcircuit consists of a parallel connection of a resistor and a CPE. The values for the different elements are collected in Tables I and II and the fits are shown in Figs. 5 and 6 as solid lines through the data points. The characteristic time constants are calculated using $\tau=R Q^{(1 / n)}$ and are included in Tables I and II.

One of the CPE's behaves as a true capacitance, i.e., $n$ $\approx 1$, and correlates to the geometrical capacitance. IMPS spectra of cells with an extra series resistor $R_{S}$ show two time constants $\tau_{1}$ and $\tau_{2}$, which are collected in Table I and plotted as a function of $R_{s}$ in Fig. 7. $Q_{1}$ is determined by the geometry of the cell and $R_{1}$ by the resistance of the ITO plus the external resistor; $\tau_{1}$ is the $R C$ time of the cell. By connecting a resistor in series to the cell, the associated $R C$ time should increase linearly as is indeed observed (Fig. 7). The intercept with the $R_{s}$ axis is close to zero, which shows that the internal resistance of the cells is small.

The second CPE in Table I has a phase factor $n$ that varies between 0.43 and 0.68 , which is typical for diffusionlike processes. Hole transport from the $\mathrm{TiO}_{2} / \mathrm{ZnTCPP}$ interface 


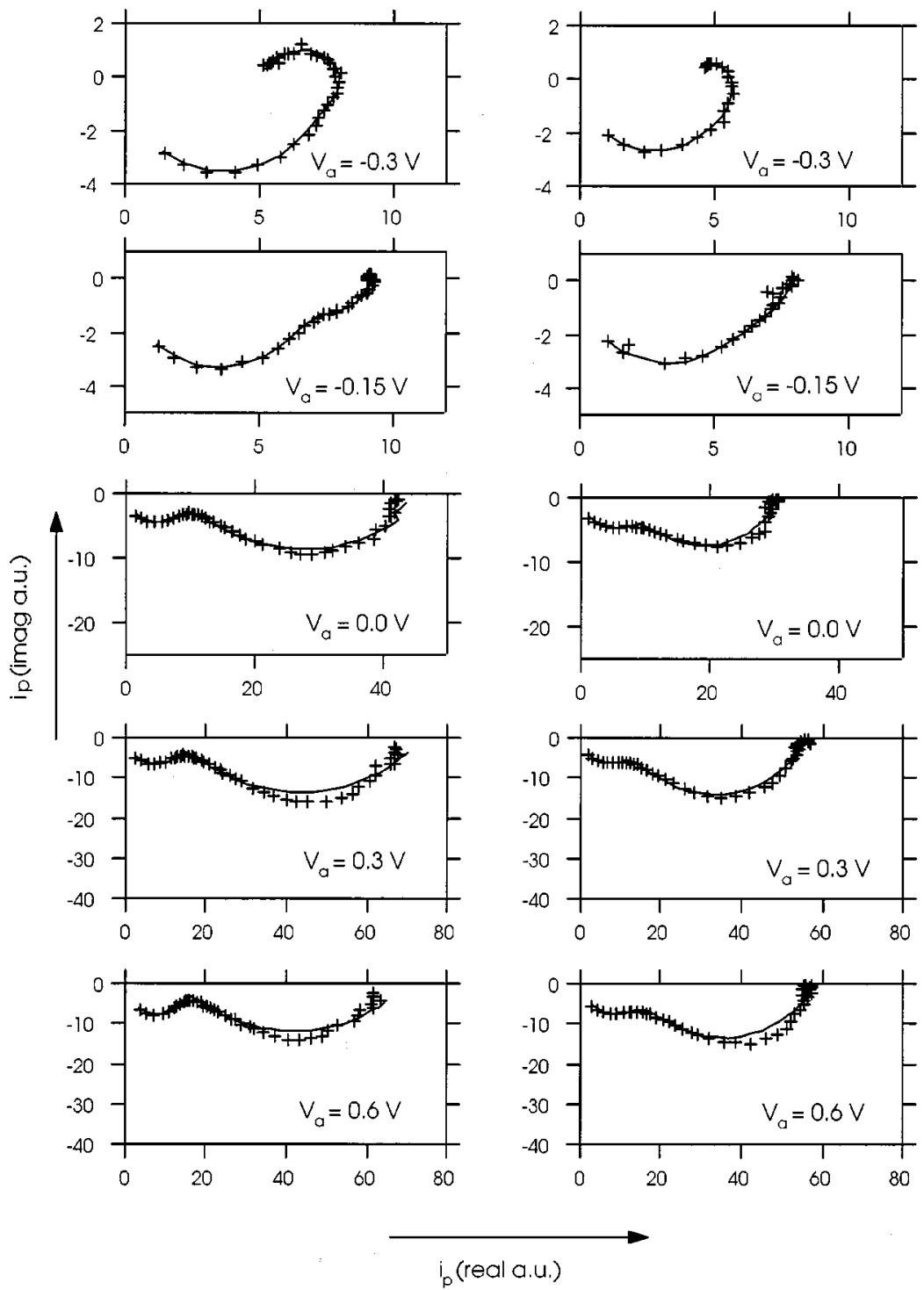

FIG. 6. IMPS spectra (+) of $\mathrm{ITO} / \mathrm{TiO}_{2}(200$ $\mathrm{nm}) / \mathrm{ZnTCPP}(45 \mathrm{~nm}) / \mathrm{Hg}$ cells with applied bias voltages in the dark (left) and with $100-\mathrm{mW} \mathrm{cm}^{-2}$ irradiation (right). The fits of the equivalent circuit to the IMPS spectra are excellent and are represented by the solid lines drawn through the data points. The fit parameters are collected in Table II. A $100 \Omega$ resistor is used to measure the current.

across the porphyrin film to the metal back contact is associated with this CPE element. The corresponding $\tau_{2}$ in Fig. 7 shows a decrease by more than two orders of magnitude upon increasing $R_{s}$. Accordingly, a higher series resistance yields faster hole transport. This is surprising, since the photocurrent admittance at low frequencies (the external quantum efficiency) does not correlate strongly with the external series resistance. Figure 8 shows $\tau_{1}$ and $\tau_{2}$ as a function of

TABLE I. Fit parameters for IMPS spectra using different series resistors $R_{s}$, where $R_{1}$ and $Q_{1}$ are associated with the geometry of the cell, and $R_{2}$ and $C_{2}$ are associated with the transport of holes through ZnTCPP films.

\begin{tabular}{llllllll}
\hline \hline $\begin{array}{l}R_{s} \\
(\Omega)\end{array}$ & $\begin{array}{l}R_{1} \\
(\Omega)\end{array}$ & $\begin{array}{l}Q_{1} \\
\left(\times 10^{-5}\right)\end{array}$ & $n_{1}$ & $\begin{array}{l}R_{2} \\
(\Omega)\end{array}$ & $Q_{2}$ & $n_{2}$ & $\begin{array}{l}x^{2} \\
\left(\times 10^{-4}\right)\end{array}$ \\
\hline 100 & $18 \times 10^{-3}$ & 5.1 & 1 & 0.068 & 0.45 & 0.43 & 4.1 \\
200 & $43 \times 10^{-3}$ & 3.6 & 1 & 0.16 & 0.18 & 0.47 & 4.0 \\
500 & 0.11 & 2.7 & 1 & 0.35 & 0.08 & 0.44 & 2.7 \\
1000 & 0.21 & 2.8 & 1 & 0.61 & 0.041 & 0.46 & 3.0 \\
2000 & 0.63 & 1.9 & 1 & 1.39 & 0.014 & 0.5 & 2.7 \\
5000 & 2.0 & 1.5 & 1 & 3.1 & 0.004 & 0.57 & 2.2 \\
10000 & 4.8 & 1.2 & 1 & 5.4 & 0.0017 & 0.64 & 2.2 \\
20000 & 10 & 1.1 & 1 & 9.2 & 0.0011 & 0.68 & 1.2 \\
\hline \hline
\end{tabular}


TABLE II. Fit parameters for IMPS spectra recorded at different applied bias voltages with $100 \mathrm{~mW} \mathrm{~cm}^{-2}$ tungsten-halogen light and in the dark.

\begin{tabular}{|c|c|c|c|c|c|c|c|}
\hline $\begin{array}{l}V_{a} \\
(\mathrm{~V})\end{array}$ & $\begin{array}{l}R_{1} \\
(\mathrm{~m} \Omega)\end{array}$ & $Q_{1}$ & $n_{1}$ & $\begin{array}{l}R_{2} \\
(\mathrm{~m} \Omega)\end{array}$ & $Q_{2}$ & $n_{2}$ & $\begin{array}{l}x^{2} \\
\left(\times 10^{-4}\right)\end{array}$ \\
\hline \multicolumn{8}{|c|}{ Light background } \\
\hline 0.6 & 13 & 0.00031 & 0.94 & 45 & 0.081 & 0.68 & 13.5 \\
\hline 0.3 & 11 & 0.000405 & 0.95 & 46 & 0.082 & 0.7 & 10 \\
\hline 0 & 8.3 & 0.00052 & 0.95 & 22 & 0.066 & 0.74 & 8.4 \\
\hline-0.15 & 5.3 & 0.0004 & 0.99 & 2.6 & 0.077 & 0.75 & 2.8 \\
\hline-0.3 & 4.6 & 0.00034 & 1 & 3.2 & 0.041 & 0.61 & 4.6 \\
\hline \multicolumn{8}{|c|}{ Dark background } \\
\hline 0.6 & 14 & 0.0002 & 0.99 & 56 & 1.15 & 0.51 & 20 \\
\hline 0.3 & 13 & 0.00037 & 0.93 & 61 & 0.87 & 0.53 & 16 \\
\hline 0 & 8.3 & 0.00041 & 0.96 & 39 & 1.07 & 0.53 & 8.4 \\
\hline-0.15 & 6.6 & 0.00041 & 0.97 & 2.6 & 0.11 & 0.82 & 3.2 \\
\hline-0.3 & 6.9 & 0.00029 & 0.99 & 2.2 & 0.17 & 0.46 & 4.9 \\
\hline
\end{tabular}

the bias voltage. Charging the cell is associated with $\tau_{1}$, being the product of the series resistance and the geometrical capacitance. This time constant is a property of the device and is only little affected by the potential or by background irradiation (see Fig. 4). The second time constant $\tau_{2}$ is independent of potential between 0.0 and $0.6 \mathrm{~V}$. Hole transport through the 32-nm ZnTCPP film takes about $70 \mathrm{~ms}$ irrespective of the presence of an electrical field. Even an internal electric field of approximately $0.2 \mathrm{MV} / \mathrm{cm}$ does not speed up the transport of holes. It is clear that diffusion rather than drift determines hole transport through 32-nm porphyrin films, in agreement with the observed phase factor $n$ $=\sim 0.5$. In contrast, a small forward bias reduces $\tau_{2}$ by two orders of magnitude. Similar observations have been made with time-resolved photocurrent measurements at different applied potentials. ${ }^{17,18,23}$ Furthermore, when background illumination is applied, $\tau_{2}$ also reduces by two orders of magnitude when a reserse bias between 0 and $0.6 \mathrm{~V}$ is applied. Irradiation has much lesser effect on $\tau_{2}$ when a forward bias is applied.

\section{Trap-assisted hole diffusion}

Application of a small forward-bias voltage imposes huge differences in transit time $\tau_{2}$. This behavior is also reported

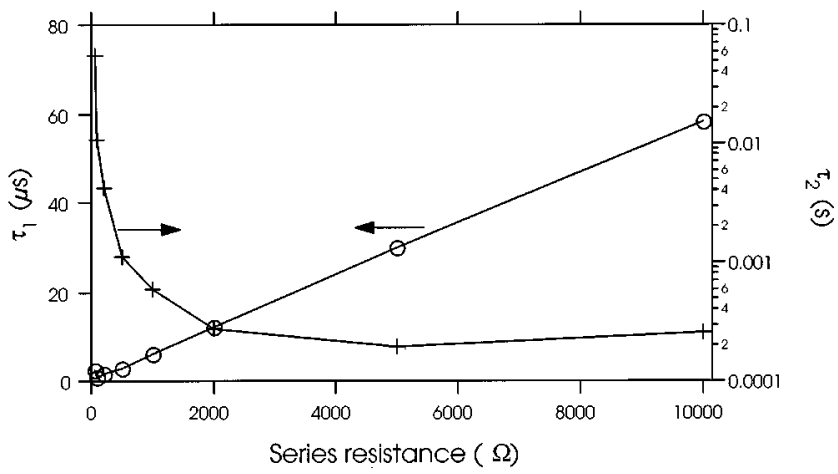

FIG. 7. Dependence of time constants associated with charging of the cell (geometrical $R C$ time), $\tau_{1}(\bigcirc)$, and the transit time, $\tau_{2}(+)$, as a function of the externally connected series resistance. for electron transport in nanoporous anatase $\mathrm{TiO}_{2}$ electrodes ${ }^{10}$ and is related to the capacity of these electrodes to store electrons. With a small forward bias, deep electron traps are filled that allow the electrons to hop along shallow traps, which reduce the transit time. ${ }^{42}$ Here a similar situation exists. The ZnTCPP film is a collection of porphyrin molecules randomly distributed in the amorphous film. The central zinc atoms can have different ligands such as a water and oxygen. The energy level of the HOMO is modulated by these local distortions. Energetic fluctuation of the HOMO can be envisaged as a hole trap since holes captured in deep energy states are relatively immobile. An estimation for the amount of energetic disorder can be obtained by comparing the absorption spectrum of dissolved porphyrin molecules ( 2 $\mu \mathrm{M})$ with that of the films. The absorption spectrum of the solution resembles that of porphyrin monomers. ${ }^{8}$ In Fig. 3(b) these two absorption spectra are shown. The absorption spectrum of ZnTCPP on quartz is broadened and shows a redshift of approximately $25 \mathrm{~nm}$. This is indicative for the presence of intermolecular interactions in amorphous films. ${ }^{43}$ The onset of the lowest absorption band shifts from 625 to 650 $\mathrm{nm}$, which corresponds to an energy difference of $76 \mathrm{meV}$.

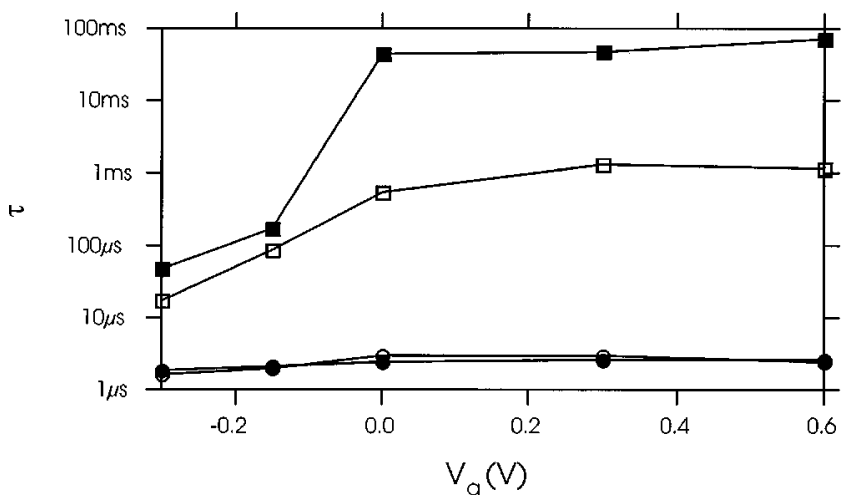

FIG. 8. Dependence of time constants associated with the charging of the cell, $\tau_{1}(\bigcirc)$, and with the transit time, $\tau_{2}(\square)$, as a function of the applied bias; in the dark (filled markers) and in the presence of additional bias light (open markers). 

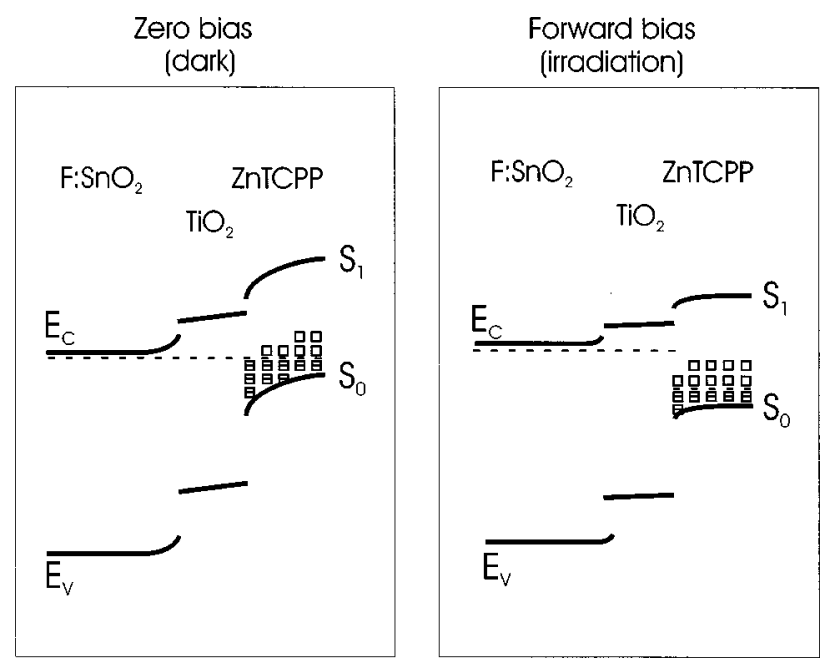

FIG. 9. Schematic presentation of the trap-filling statistics as a function of the applied bias voltage. Applying a forward bias reduces the band bending, which leads to filling of deep hole traps in the space-charge region. Under irradiation photodoping occurs due to electron transfer from photoexcited ZnTCPP molecules to incorporated oxygen. An increase in the acceptor density $N_{A}$ is the result. The (quasi-) Fermi level moves towards the HOMO and again deep hole traps become filled.

In the cells, excitons are dissociated at the $\mathrm{TiO}_{2} / \mathrm{ZnTCPP}$ interface. The holes remain in ZnTCPP and diffuse slowly via traps towards the back contact. With a forward bias the band bending in the ZnTCPP film is reduced. In the spacecharge region the Fermi level is lowered towards the HOMO, which fills up deep energy states with holes. Hole transport now takes place via the shallow traps, which speed up the diffusion process significantly. This process is schematically shown in Fig. 9, which represents the situation under zerobias voltage (short circuit) and when a forward-bias voltage is applied.

When light shines onto the $\mathrm{TiO}_{2}$ /ZnTCPP films, the acceptor density increases by a factor of 3 [see Fig. 4(b)], which is accompanied by a strong reduction of the transit time (Fig. 8). The concept of photoinduced electron transfer from excited porphyrin to oxygen has been discussed above and it is this type of charging that also explains the enhanced hole mobility. Generation of immobile negative charge is accompanied by an increase of the hole concentration to maintain local charge neutrality. By shining light, the band bending in ZnTCPP is reduced and the (quasi-) Fermi-energy level shifts down towards the HOMO. Deep hole traps are filled, which speeds up hole diffusion through the film. Irradiating the sample is electronically almost equivalent to applying a forward bias. In both cases the band bending is reduced and the Fermi level shifts down towards the HOMO. Moreover, by shining light the acceptor density in ZnTCPP increased by a factor of 3 , which gives a small additional shift of the Fermi level. Accordingly, Fig. 9 can also serve to illustrate the effect of irradiation.

Assuming that hole transport is governed by diffusion, the transit time can be used to calculate the diffusion coefficient $D$ via

$$
D=\frac{\lambda^{2}}{6 \tau_{2}}
$$

where $\lambda$ is the thickness of the film. In the dark at zero bias, $D=7 \times 10^{-11} \mathrm{~cm}^{2} \mathrm{~s}^{-1}$, which increases to $D=2$ $\times 10^{-7} \mathrm{~cm}^{2} \mathrm{~s}^{-1}$ when either forward bias or background irradiation is applied. The lower value agrees well with electric and electrochemical measurements on similar porphyrin thin films. ${ }^{16,21,26,44}$

\section{CONCLUSIONS}

$\mathrm{ITO} / \mathrm{TiO}_{2} / \mathrm{ZnTCPP} / \mathrm{Hg}$ cells can be described as $n^{+}-n-p$ heterojunction as follows from $i-V$ and $C-V$ measurements. Without optical irradiation, the donor density of $\mathrm{TiO}_{2}$ is $N_{D}$ $=2 \times 10^{16} \mathrm{~cm}^{-3}$ and the acceptor density of ZnTCPP is $N_{A}$ $=4.0 \times 10^{17} \mathrm{~cm}^{-3}$. By applying background light, $N_{A}$ increases to $1.3 \times 10^{18} \mathrm{~cm}^{-3}$. The increase of $N_{A}$ is due to electron transfer from photoexcited porphyrin molecules to incorporated oxygen. Accordingly, the depletion region inside the porphyrin films is controlled by the applied potential and by the presence of light. In the present study, experiments have been performed in air, i.e., an oxygen partial pressure of 0.2 bar. Since oxygen acts as dopant, practical devices should be prepared in well-controlled conditions to improve their reliability. This, however, lies beyond the scope of the present study.

IMPS spectra indicate that the transport of holes is not governed by the electric field, since the transit time is insensitive to the applied reverse bias but is driven by diffusion. Applying either a forward potential or background light, the transit time reduces more than two orders of magnitude. This reduction is caused by a shift of the (quasi-) Fermi level towards the HOMO. Deep hole traps are filled and hole migration occurs via shallow traps, which speeds up hole migration significantly.

The hole traps originate from local fluctuations in the HOMO levels caused by random distribution of porphyrin molecules in amorphous films. The presence of deep traps is detrimental for fast hole conduction and must be avoided. An increase of the hole diffusivity from $7 \times 10^{-11}$ to $2 \times 10^{-7} \mathrm{~cm}^{2} \mathrm{~s}^{-1}$ is realized by applying a forward bias or by shining light. Mobilities of the order of $5 \times 10^{-4} \mathrm{~cm}^{2} \mathrm{~V}^{-1} \mathrm{~s}^{-1}$ have been found for columnar stacks of porphyrins,${ }^{20}$ which demonstrates that in this class of materials, fast charge carrier transport is, in principle, feasible. Molecular self-assembly is therefore a prerequisite when high mobilities are desired, as is the case in organic solar cells.

\section{ACKNOWLEDGMENT}

The authors wish to thank the Dutch Agency for Energy and the Environment (NOVEM) for their financial support. 
${ }^{1}$ C. D. Jaeger, F. F. Fan, and A. J. Bard, J. Am. Chem. Soc. 102, 2592 (1980).

${ }^{2}$ A. Giraudeau, F. F. Fan, and A. J. Bard, J. Am. Chem. Soc. 102, 5137 (1980).

${ }^{3}$ B. O'Regan and M. Grätzel, Nature (London) 334, 737 (1991).

${ }^{4}$ K. Tennakone, G. Kumara, K. G. U. Wijayantha, I. R. M. Kottegoda, V. P. S. Perera, and G. Aponsu, J. Photochem. Photobiol., A 108, 175 (1997).

${ }^{5}$ J. Hagen, W. Schaffrath, P. Otschik, R. Fink, A. Bacher, H. W. Schmidt, and D. Haarer, Synth. Met. 89, 215 (1997).

${ }^{6}$ U. Bach, D. Lupo, P. Comte, J. E. Moser, F. Weissortel, J. Salbeck, H. Spreitzer, and M. Grätzel, Nature (London) 395, 583 (1998).

${ }^{7}$ P. A. van Hal, M. P. T. Christiaans, M. M. Wienk, J. M. Kroon, and R. A. J. Janssen, J. Phys. Chem. B 103, 4352 (1999).

${ }^{8}$ K. Kalyanasundaram, N. Vlachopoulos, V. Krishan, A. Monnier, and M. Grätzel, J. Phys. Chem. 91, 2342 (1987).

${ }^{9}$ A. Kay and M. Grätzel, J. Phys. Chem. 97, 6272 (1993).

${ }^{10}$ G. K. Boschloo and A. Goossens, J. Phys. Chem. 100, 19489 (1996).

${ }^{11}$ J. Wienke, T. J. Schaafsma, and A. Goossens, J. Phys. Chem. B 103, 2702 (1999).

${ }^{12}$ D. Schlettwein and N. R. Armstrong, J. Phys. Chem. 98, 11771 (1994).

${ }^{13}$ D. Schlettwein, N. I. Jaeger, and D. Woehrle, Ber. Bunsenges. Phys. Chem. 95, 1526 (1991).

${ }^{14}$ D. Wöhrle, L. Kreienhoop, G. Schnurpfeil, J. Elbe, B. Tennigkeit, S. Hiller, and D. Schlettwein, J. Mater. Chem. 5, 1819 (1995).

${ }^{15}$ T. J. Savenije, E. Moons, G. K. Boschloo, A. Goossens, and T. J. Schaafsma, Phys. Rev. B 55, 9685 (1997).

${ }^{16}$ S. Nespurek, R. H. G. Hart, J. S. Bonham, and L. E. Lyons, Aust. J. Chem. 38, 1061 (1985).

${ }^{17}$ T. Nagamura, K. Matano, and T. Ogawa, J. Phys. Chem. 91, 2019 (1987).

${ }^{18}$ T. Nagamura, S. Kamata, K. Sakai, K. Matano, and T. Ogawa, Thin Solid Films 179, 293 (1989).

${ }^{19}$ W. A. Nevin and G. A. Chamberlain, J. Chem. Soc., Faraday Trans. 85, 1729 (1989).

${ }^{20}$ P. G. Schouten, J. M. Warman, M. P. De haas, M. A. Fox, and H. L. Pan, Nature (London) 353, 736 (1991).

${ }^{21}$ M. F. Lawrence, Z. Huang, C. H. Langford, and I. Ordonez, J. Phys. Chem. 97, 944 (1993).

${ }^{22}$ A. Ioannidis, M. F. Lawrence, H. Kassi, R. Cote, J. P. Dodelet, and R. M. Leblanc, Chem. Phys. Lett. 205, 46 (1993).
${ }^{23}$ M. A. Fox, H. L. Pan, W. E. Jones, and D. Melamed, J. Phys. Chem. 99, 11523 (1995).

${ }^{24}$ C. Nasr, S. Hotchandani, H. Kassi, S. Nsengiyumva, and R. M. Leblanc, Sol. Energy Mater. Sol. Cells 36, 261 (1995).

${ }^{25}$ T. Taleb, C. Nasr, S. Hotchandani, and R. M. Leblanc, J. Appl. Phys. 79, 1701 (1996).

${ }^{26}$ T. J. Savenije, R. B. M. Koehorst, and T. J. Schaafsma, J. Phys. Chem. B 101, 720 (1997).

${ }^{27}$ M. A. Fox, J. V. Grant, D. Melamed, T. Torimoto, C. Y. Liu, and A. J. Bard, Chem. Mater. 10, 1771 (1998).

${ }^{28}$ S. M. Sze, Physics of Semiconductor Devices, 2nd ed. (Wiley, New York, 1981).

${ }^{29}$ I. H. Campbell, D. L. Smith, and J. P. Ferraris, Appl. Phys. Lett. 66, 3030 (1995).

${ }^{30}$ S. Gunster, S. Siebentritt, J. Elbe, L. Kreienhoop, B. Tennigkeit, D. Wohrle, R. Memming, and D. Meissner, Mol. Cryst. Liq. Cryst. Sci. Technol., Sect. A 218, 641 (1992).

${ }^{31}$ B. A. Gregg and Y. I. Kim, J. Phys. Chem. 98, 2412 (1994).

${ }^{32}$ T. J. Savenije, R. B. M. Koehorst, and T. J. Schaafsma, Chem. Phys. Lett. 244, 363 (1995).

${ }^{33}$ H. R. Kerp and E. E. van Faassen, Phys. Chem. Chem. Phys. 1, 1761 (1999).

${ }^{34}$ L. M. Peter, Chem. Rev. 90, 753 (1990).

${ }^{35}$ G. Franco, J. Gehring, L. M. Peter, E. A. Ponomarev, and I. Uhlendorf, J. Phys. Chem. B 103, 692 (1999).

${ }^{36}$ G. K. Boschloo, A. Goossens, and J. Schoonman, J. Electrochem. Soc. 144, 1723 (1997).

${ }^{37}$ C. H. M. Marée, S. Roosendaal, T. J. Savenije, R. E. I. Schropp, T. J. Schaafsma, and F. H. P. M. Habraken, J. Appl. Phys. 80, 3381 (1996).

${ }^{38}$ W. A. Nevin and G. A. Chamberlain, J. Appl. Phys. 69, 4324 (1991).

${ }^{39}$ T. J. Savenije, J. M. Warman, and A. Goossens, Chem. Phys. Lett. 287, 148 (1998).

${ }^{40}$ R. van deKrol, A. Goossens, and J. Schoonman, J. Electrochem. Soc. 144, 1723 (1997).

${ }^{41}$ J. Simon and J. J. Andre, Molecular Semiconductors (SpringerVerlag, Berlin, 1985).

${ }^{42}$ K. Schwarzburg and F. Willig, Appl. Phys. Lett. 58, 2520 (1991).

${ }^{43}$ R. G. Stomphorst, T. J. Schaafsma, and G. van der Zwan, J. Phys. Chem. A 105, 4226 (2001).

${ }^{44}$ B. A. White and R. W. Murray, J. Am. Chem. Soc. 109, 2576 (1987). 